\title{
Bradycardia and cardiac arrest during laparoscopic surgery
}

\begin{abstract}
Bradycardia after gas insufflation during laparoscopic surgery should be taken seriously as it maybe develop a cardiac arrest. A 25years old healthy male patient with no medical history or previous anesthesia was scheduled for laparoscopic cholecystectomy. Suffered from unexpected intra-operative cardiac arrest after Bradycardia few minutes after $\mathrm{CO}_{2}$ pneumoperitoneum insufflation. It was immediately corrected with quick response, IV Atropine administration, and deflation of pneumoperitoneum. The patient underwent a successful surgery at the same setting. This article identify successful management strategies in the possible prevention and treatment of cardiac arrest after Bradycardia during laparoscopic surgeries.
\end{abstract}

Volume 8 Issue I - 2017

\author{
Nahed Makkieh \\ Master Degree in Anesthesia, Damascus University, Qatar \\ Correspondence: Nahed Makkieh, Master Degree in \\ Anesthesia, Damascus University, Ministry of High Education, \\ Syrian Bord of Anaesthesia, Ministry of health, Qatar, \\ Email nahedmak75@yahoo.com
}

Received: January 21, 2017 | Published: January 23, 2017

\section{Introduction}

Laparoscopic surgery is widely established for a range of procedures including cholecystectomy, hernia repair, and appendectomy, and more complex surgeries like colonic resection, nephrectomy, and bariatric surgery. ${ }^{1,2}$ It has proved post-operative benefits including: reduced pain, tissue trauma, and wound size, improved post-operative pulmonary function, earlier mobility, shorter hospital admission, and reduced health costs. ${ }^{1,3}$ There are, however, specific complications to laparoscopic surgery which vascular injury, gas embolism, and cardiac arrest are the most serious. ${ }^{3}$ Although rare, such complications can be fatal and are demanding to the anesthesiologist. ${ }^{4}$

Artificial pneumoperitoneum, using carbon dioxide $\left(\mathrm{CO}_{2}\right)$ insufflation at a rate of $4-6$ liters $/ \mathrm{min}$ to a pressure of $10-15 \mathrm{mmHg}$, is essential to separate the abdominal wall from the viscera, which improves visualization to the surgeon. ${ }^{5}$ The capnoperitoneum is maintained by a constant gas flow of $200-400 \mathrm{ml} / \mathrm{min}$. carbon dioxide allows the use of diathermy or laser as it is a non-combustible gas.

This case was compared with other articles to specify the cause of intra-operative Bradycardia and cardiac arrest after gas insufflation. Serious intraoperative cardiac arrest is reported to occur between 2 and 40 per 100000 laparoscopic cases. ${ }^{6}$ Vagal response after artificial pneumoperitoneum is considered the most commonly reason for Bradycardia, this may happen in fit and healthy patients with no significant medical history. ${ }^{7}$ The management of Bradycardia should be effective and definitive as it maybe an early warning for cardiac arrest, this management includes: IV Atropine administration, and deflation of pneumoperitoneum. ${ }^{3}$ If Bradycardia developed to cardiac arrest the management includes: IV Atropine administration, pneumoperitoneum deflation, CPR.

\section{Case report}

A 25years old male patient was admitted to our hospital for laparoscopic cholecystectomy. The patient pre-operative assessment in the anesthesia clinic was normal, no medical history, no previous surgeries, smoker, pre-operative fasting blood sugar, serum electrolytes, urea, and creatinine were all within normal limits. He had a normal chest $x$-ray. In the operating room standard monitors (ECG, pulse oximetry, non invasive blood pressure (NIBP)) were placed. Initial blood pressure BP and heart rate were $128 / 65 \mathrm{mmHg}$, $74 \mathrm{bpm}$ respectively. Induction through a proper IV line started with fentanyl $(100 \mathrm{mcg})$, propofol $(2 \mathrm{mg} / \mathrm{kg})$, and rocuronium $(0,6 \mathrm{mg} / \mathrm{kg})$, intubation done with $8,0 \mathrm{~mm}$ endotracheal tube. Ventilator settings: pressure controlled ventilation $15 \mathrm{mmHg}$, fresh gas flow rate of 2liters/ min, and respiratory rate was regulated to maintain a normal end-tidal $\mathrm{CO}_{2}\left(\mathrm{EtCO}_{2}\right)$.

The primary trocar inserted and gas (carbon dioxide) insufflation started in the peritoneal cavity. Sudden sinus Bradycardia was noticed after few minutes, Atropine $0,5 \mathrm{mg}$ given intravenously (IV) but it rapidly progressed to sever Bradycardia, Atropine $0,5 \mathrm{mg}$ IV given again, Inhalational agent ceased, and oxygen given by endotracheal tube at a rate of 6-8liters/min. The surgeon was asked to desufflate the abdomen immediately, even though, stand still happened, chest compression started and a third Atropine $0,5 \mathrm{mg}$ (IV) given, at the same moment heart beats started to appear on monitor and heart rate became 80-90 bpm. $\mathrm{O}_{2} \mathrm{Sat}, \mathrm{EtcO}_{2}$, Bp were normal all the time. The patient hemodynamic status was stable and the surgery done at the same settings. At the end of the procedure, neuromuscular blocked was reversed with Neostigmine plus Atropine and the trachea extubated. The post-operative investigations were performed, ECG was normal.

\section{Discussion}

Laparoscopic surgery involves insufflation of a gas (usually carbon dioxide) into the peritoneal cavity producing a pneumoperitoneum. This causes an increase in intra-abdominal pressure (IAP) ${ }^{8}$ The raised intra-abdominal pressure of the pneumoperitoneum, alteration in the patient's position and effects of carbon dioxide absorption cause changes in physiology, especially within the cardiovascular and respiratory systems. These changes, as well as direct effects of gas insufflation, may have significant effects on the patient.

Bradycardia during gas insufflation is not necessarily a benign event, ${ }^{3,7}$ it maybe an early warning sign for possible impending and unexpected cardiac arrest. Unpredicted cardiac arrest in routine fit patient without any risk factors is considered distressing for the anaesthesiologist. The causes include: $\mathrm{CO}_{2}$ pulmonary embolism, vagal responses, hypercarbia, and reduced cardiac preload.

This article will discuss the possible cause of Bradycardia related to this case. Peritoneal triggered vagal responses: Rapid peritoneal stretching with pneumoperitoneum can lead to significant vagal stimulation resulting in sever sinus Bradycardia, nodal rhythm, and occasional asystole in a young healthy patient, recovery of this 
situation achieved with release of pneumoperitoneum, and treat with vagolytics. Insufflation related Bradycardia is reported $28 \%$ of laparoscopic surgeries; the treatment using deflation of the gas, Atropine administration is the best. ${ }^{9}$

Management of cardiac arrest during insufflation is a combination of Atropine administration, cardiopulmonary resuscitation (CPR), deflation of pneumoperitoneum, Adrenaline administration, and repositioning into the supine position. All the cases mentioned in other articles with Bradycardia progressed to cardiac arrest in young healthy patient, were associated with effective reversal of cardiac arrest with no reported mortality.

\section{Conclusion}

Although Bradycardia is a common occurrence during laparoscopy, it should be considered a critical clinical mark for cardiac arrest. It usually arises in response to gas insufflation as a vagal mediated physiologic response, and is benign in the majority of cases. Prediction which Bradycardia episodes will go to cardiac arrest remains unclear. Deflation of pneumoperitoneum and Atropine administration are effective in reversing Bradycardia and possible avoid progression to cardiac arrest. Peri-insufflation Bradycardia should be a significant warning for both surgeons and anesthetists.

\section{Acknowledgments}

None.

\section{Conflicts of interest}

Author declares there are no conflicts of interest.

\section{Funding}

None.

\section{References}

1. Oxford Handbook of Anesthesia (2012).

2. Clinical anesthesiology (LANGE).

3. Jonathan Yong, Peter Hibbert, William B Runciman, et al. Bradycardia as an early warning sign for cardiac arrest during routine laparoscopic surgery. Int J Qual Health Care (2015). 2015;27(6):473-478.

4. Gautam B, Shrestha BR. Cardiac arrest during laparoscopic cholecystectomy. Kathmandu Univ MedJ(KUMJ) . 2009;7(27):280-288.

5. Jung KT, Kim SH, Kim JW, et al. Bradycardia during laparoscopic surgery. Korean J Anesthesiol. 2013;65(3):276-277.

6. Bradycardia as an early warning sign for cardiac arrest during routine laparoscopic surgery: Medscape. 2016.

7. Reed DN Jr, Duff JL. Persistent occurrence of Bradycardia during laparoscopic cholecystectomies in low risk patients. Dig Surg. 2000;17(5):513-517.

8. Laparoscopic abdominal surgery, Mandy Perrin, oxford journals.

9. Aghamohammadi H, Mehrabi S, Mohammad Ali Beigi F. Prevention of bradycardia by atropine sulfate during urological laparoscopic surgery: a randomized controlled trial. Urol J. 2009;6(2):92-95. 